\title{
Low-Dose Chemomodulation and Cancer Vaccines
}

\section{Michael R Shurin* and Anton A Keskinov}

Departments of Pathology and Immunology, University of Pittsburgh Medical Center and University of Pittsburgh Cancer Institute, Pittsburgh, PA, USA

Keywords: Immunotherapy; Chemotherapy; Chemomodulation; Tumor microenvironment; Antitumor immunity

Recent advances in clinical and experimental oncology and immunology have markedly expanded our understanding of the role of the immune system response to precancerous cells, their malignization and expansion, as well as to tumor formation, growth and progression. These achievements resulted in development of improved and novel therapeutic modalities, which involve the treatment of cancer by modification of the host-tumor relationship. For instance, the main goal of antitumor immunotherapy is to provide either passive or active long-lasting immunity against malignancies by controlling the immune system responsiveness to tumors. Much of the success of anticancer immunotherapeutics has come from monoclonal antibodies delivering their biological activities either through immune-related effector functions, specific delivering of anticancer agents or by inhibiting dysregulated ligand-receptor interactions. However, despite their significant clinical success, antibodies have several well-known limitations, including expensive manufacturing, limited penetration into the tumor mass and some potential undesired systemic effects. Therefore, in addition to the therapeutic antibodies, immunomodulators (e.g., TLR ligands, thalidomide and its analogues, Lactoferrin), cytokines/chemokines/growth factors, cellular immunotherapy and vaccines have increasingly become thriving therapeutic agents for the treatment of solid and hematological malignancies in preclinical models, clinical trials and even clinical practice.

Although immunotherapy has emerged as an alternative option for the treatment of cancer patients in the last decades, the presented evidence indicates that both active and adoptive immunotherapeutic strategies are somewhat effective against small tumor burdens, but are generally insufficient to eliminate the disease in patients with advanced stage cancer, despite induction of tumor-specific immune responses [1]. Furthermore, while vaccine approaches have had some clinical achievement, most cancer vaccines fail to induce objective tumor shrinkage in patients. New modalities have centered on a group of molecules known as immune checkpoints, since their function is to contain or diminish potentially exuberant reactions. Antibody-based blocking of immune checkpoint molecules has emerged as a viable clinical approach that mediates tumor shrinkage in several cancer types. In addition to being part of the current treatment armamentarium for metastatic melanoma, immune checkpoint blockade is currently undergoing phase III testing in several cancer types [2]. Targeting co-inhibitory and co-stimulatory receptors with immunostimulatory antibodies has also shown clinical promise and its combined use with vaccines is a promising new approach of immunotherapy for cancer [1].

Advances in our understanding of the primary mechanisms of immune and cancerous cell interactions and the functioning of the tumor immunoenvironment have also provided the platform for combining cancer vaccines with chemotherapies neutralizing, to some extent, tumor-induced suppressive network and demonstrating clinical efficacy. A growing body of clinical data suggests that despite the great specificity that can be achieved with immunotherapy and the potency of cytotoxic anticancer agents, neither of these two modalities by itself has been sufficient to eradicate the disease [3]. Therefore, the combination of chemotherapy and immunotherapy is no longer considered incompatible, because of the emerging insight that certain chemotherapy-based cancer treatments may activate the immune system against the tumor through several molecular and cellular mechanisms [4]. Indeed, certain chemotherapeutic agents have shown immunomodulatory activities, and several combined approaches have already been attempted [5].

Chemotherapeutics and immunotherapy may thus be synergistic and improve the clinical response in cancer. For instance, chemotherapy has been proven to enhance the efficacy of vaccination $[6,7]$ and to favor the activity of adoptively transferred tumor-specific $\mathrm{T}$ cells or dendritic cells (DC) [8,9]. A potential efficacy of combining neo-adjuvant chemotherapy and immunotherapy with PBMC and IL-2 in the treatment of NSCLC patients has been recently reported [10]. Furthermore, when combined with CTLA-4 (a cytotoxic T lymphocyte antigen-4) blockade, ixabepilone, etoposide and gemcitabine elicited prolonged antitumor effects in several murine models with induction of a memory immune response [11]. Other data support the combinatorial use of immunomodulators, such as ipilimumab (anti-CTLA-4 antibody), with traditional chemotherapy regimens to improve SCLC patient outcomes and potentially sustain the effect from chemotherapeutic induction [12]. Synergy is mediated by diverse mechanisms, including preferential depletion of regulatory $\mathrm{T}$ cells, liberation of homeostatic or inflammatory cytokines and enhanced immunogenicity of chemotherapy-treated tumors. Thus, chemotherapy may favor tumor cell death, and by that enhance tumor-antigen crosspresentation in vivo. Drug-induced myelosuppression may induce the production of cytokines favoring homeostatic proliferation, and/or ablate immunosuppression mechanisms [3]. Furthermore, the recently reported synergy between monoclonal antibodies and chemotherapy or peptide vaccination is based upon the induction of endogenous humoral and cellular immune responses. This would suggest that monoclonal antibodies may not only provide passive immunotherapy but can also promote tumor-specific active immunity [1].

Given a growing enthusiasm to combining chemotherapeutic and immunomodulating agents for cancer treatment, it is important to notice that only a few cytotoxic drugs were proven to improve the therapeutic efficacy of cancer vaccines, while the majority of conventional chemotherapies are still immunosuppressive [5]. For

*Corresponding author: Michael R. Shurin, UPMC, Pathology, Scaife Hall S735, 3550 Terrace St, Pittsburgh, PA 15261, USA, Tel: 412-648-9831; Fax: 412-6477741; E-mail: shurinmr@upmc.edu

Received December 12, 2013; Accepted December 29, 2013; Published January 02, 2014

Citation: Shurin MR, Keskinov AA (2014) Low-Dose Chemomodulation and Cancer Vaccines. J Vaccines Vaccin 5: e126. doi: 10.4172/2157-7560.1000e126

Copyright: (c) 2014 Shurin MR, et al. This is an open-access article distributed under the terms of the Creative Commons Attribution License, which permits unrestricted use, distribution, and reproduction in any medium, provided the original author and source are credited. 
instance, to examine the impact of clinically relevant doses of alkylating chemotherapeutics (temozolomide and cyclophosphamide) on cancer vaccines, Litterman et al. used controlled animal experiments that minimized the numerous complicating factors encountered in human patients [13]. The results clearly demonstrated that alkylating chemotherapy has a long-lasting antiproliferative effect on lymphocytes, and this effect leads to profound impairment of adaptive immune responses to cancer vaccines [14]. Furthermore, the immunomodulating effects of chemotherapeutic agents might be quite complex [15]. For instance, evaluation of two clinically used chemotherapeutic agents, gemcitabine and 5-fluorouracil, known to deplete protumorigenic myeloid-derived suppressor cells (MDSC), revealed that they may also activate the inflammasomes in MDSC, leading to production of interleukin-1 $\beta$ (IL-1 $\beta$ ), which restricts anticancer immunity [16]. Next, when cyclophosphamide is administered in relatively lower doses than those routinely used in the clinical regimen, it can augment antitumor responses as it causes depletion of Treg cells [17]. In contrast, cyclophosphamide-induced MDSC accumulation was also reported by several authors [18-20], as well as low-dose cyclophosphamide-induced prevention of the development of antitumor immune responses [21]. Thus, selection of an appropriate clinically relevant combination of a chemotherapeutic drug and a cancer vaccine is far from been accepted and should be strictly based on the immunomodulating properties of a cytotoxic agent in relation to the mechanisms of the antitumor activity of a tested vaccine.

Finally, recent evaluations of potential immunomodulating activities of chemotherapeutic agents in ultra low noncytotoxic/ noncytostatic doses resulted in introduction of a new direction of research - so-called, chemomodulation or chemoimmunomodulation, - when such activities have been proven [5]. In fact, several reports revealed that certain chemotherapeutic agents could up-regulate maturation and antigen-presenting activity of murine and human DC when used in ultra-low noncytotoxic concentrations in vitro [2224]. Recent data demonstrated that ultra-low dose paclitaxel (Taxol) prevented tumor-induced polarization of conventional DC into immunosuppressive regulatory DC (regDC) in vivo and in vitro and up-regulated the antitumor potential of DC vaccines [25]. Similarly, Zhong et al. reported that a single administration of very low-dose paclitaxel synergized with DC vaccine in inhibiting lung cancer growth in mice [26]. Interestingly, paclitaxel in ultra-low concentrations was also able to support differentiation of MDSC into functionally active DC [27].

Analyzing the mechanisms of chemomodulation, Sevko et al. investigated the effect of paclitaxel applied in ultra-low, noncytotoxic doses on the efficiency of immunization of healthy mice with the peptide derived from tyrosinase related protein (TRP)-2 as a model melanoma antigen [28]. They found that administration of paclitaxel in combination with the peptide vaccination strongly increased the frequencies of TRP-2 specific T cells and was associated with a significant decrease in the levels of regulatory T cells and MDSC. Furthermore, in paclitaxel-treated mice, a significant augmentation of NK cell numbers and their ability to produce IFN- $\gamma$ were also observed [28]. Using the ret transgenic murine melanoma model, which mimics human cutaneous melanoma, the same group has tested effects of ultralow noncytotoxic dose of paclitaxel on MDSC, chronic inflammatory mediators and $\mathrm{T}$ cell activities in the tumor microenvironment in vivo [29]. Administration of paclitaxel significantly decreased accumulation and immunosuppressive activities of tumor-infiltrating MDSC without alterations of the bone marrow hematopoiesis. The production of mediators of chronic inflammation in the tumor milieu was also diminished. Importantly, reduced tumor burden and increased animal survival upon paclitaxel application was mediated by the restoration of CD8 $\mathrm{T}$ cell effector functions. This suggests that the ability of paclitaxel in a noncytotoxic dose to block the immunosuppressive potential of MDSC in vivo represents a new therapeutic strategy to down-regulate immunosuppression and chronic inflammation in the tumor microenvironment for enhancing the efficacy of concomitant anticancer therapies.

Altogether, a growing body of evidence supports a new concept that certain chemotherapeutic agents in ultra-low noncytotoxic doses may suppress tumor progression by targeting several immune cell populations in the tumor microenvironment, including DC and MDSC. New data also suggest that immunomodulating properties of certain chemotherapeutic agents in ultra low doses can be used to increase the therapeutic efficacy of cancer vaccines. However, more data are required in order to build a strong basis for developing novel clinical protocols testing the practicability and efficacy of augmentation of the antitumor properties of cancer vaccines combined with ultralow noncytotoxic doses of chemotherapeutic agents.

\section{References}

1. Baxevanis CN, Papamichail M, Perez SA (2013) Therapeutic cancer vaccines: a long and winding road to success. Expert Rev Vaccines.

2. Drake CG, Lipson EJ, Brahmer JR (2014) Breathing new life into immunotherapy: review of melanoma, lung and kidney cancer. Nat Rev Clin Oncol 11: 24-37.

3. Baxevanis CN, Perez SA, Papamichail M (2009) Combinatorial treatments including vaccines, chemotherapy and monoclonal antibodies for cancer therapy. Cancer Immunol Immunother 58: 317-324.

4. van Meir H, Kenter GG, Burggraaf J, Kroep JR, Welters MJ, et al. (2013) The Need for Improvement of the Treatment of Advanced and Metastatic Cervical Cancer, the Rationale for Combined Chemo-Immunotherapy. Anticancer Agents Med Chem.

5. Shurin MR, Naiditch H, Gutkin DW, Umansky V, Shurin GV (2012) ChemolmmunoModulation: immune regulation by the antineoplastic chemotherapeutic agents. Curr Med Chem 19: 1792-1803.

6. Kang TH, Mao CP, Lee SY, Chen A, Lee JH, et al. (2013) Chemotherapy acts as an adjuvant to convert the tumor microenvironment into a highly permissive state for vaccination-induced antitumor immunity. Cancer Res 73: 2493-2504.

7. Peng S, Lyford-Pike S, Akpeng B, Wu A, Hung CF, et al. (2013) Low-dose cyclophosphamide administered as daily or single dose enhances the antitumor effects of a therapeutic HPV vaccine. Cancer Immunol Immunother 62: $171-182$

8. Chia WK, Teo M, Wang WW, Lee B, Ang SF, et al. (2013) Adoptive T-cell Transfer and Chemotherapy in the First-line Treatment of Metastatic and/or Locally Recurrent Nasopharyngeal Carcinoma. Mol Ther.

9. Parente-Pereira AC, Whilding LM, Brewig N, van der Stegen SJ, Davies DM et al. (2013) Synergistic Chemoimmunotherapy of Epithelial Ovarian Cancer Using ErbB-Retargeted T Cells Combined with Carboplatin. J Immunol 191: 2437-2445.

10. Ratto GB, Costa R, Maineri P, Alloisio A, Piras MT, et al. (2011) Neo-adjuvant chemo/immunotherapy in the treatment of stage III (N2) non-small cell lung cancer: a phase I/II pilot study. Int J Immunopathol Pharmacol 24: 1005-1016.

11. Jure-Kunkel M, Masters G, Girit E, Dito G, Lee F, et al. (2013) Synergy between chemotherapeutic agents and CTLA-4 blockade in preclinical tumor models. Cancer Immunol Immunother 62: 1533-1545.

12. Spigel DR, Socinski MA (2013) Rationale for chemotherapy, immunotherapy, and checkpoint blockade in SCLC: beyond traditional treatment approaches. $J$ Thorac Oncol 8: 587-598.

13. Litterman AJ, Zellmer DM, Grinnen KL, Hunt MA, Dudek AZ, et al. (2013) Profound impairment of adaptive immune responses by alkylating chemotherapy. J Immunol 190: 6259-6268. 
Citation: Shurin MR, Keskinov AA (2014) Low-Dose Chemomodulation and Cancer Vaccines. J Vaccines Vaccin 5: e126. doi: 10.4172/21577560.1000 e126

14. Litterman AJ, Dudek AZ, Largaespada DA (2013) Alkylating chemotherapy may exert a uniquely deleterious effect upon neo-antigen-targeting anticancer vaccination. Oncoimmunology 2: e26294.

15. Shurin MR (2013) Dual role of immunomodulation by anticancer chemotherapy. Nat Med 19: 20-22

16. Bruchard M, Mignot G, Derangere V, Chalmin F, Chevriaux A, et al. (2013) Chemotherapy-triggered cathepsin $B$ release in myeloid-derived suppressor cells activates the NIrp3 inflammasome and promotes tumor growth. Nat Med 19: $57-64$.

17. Lutsiak ME, Semnani RT, De Pascalis R, Kashmiri SV, Schlom J, et al. (2005) Inhibition of CD4(+)25+ T regulatory cell function implicated in enhanced immune response by low-dose cyclophosphamide. Blood 105: 2862-2868.

18. Peláez B, Campillo JA, López-Asenjo JA, Subiza JL (2001) Cyclophosphamide induces the development of early myeloid cells suppressing tumor cell growth by a nitric oxide-dependent mechanism. J Immunol 166: 6608-6615.

19. Diaz-Montero CM, Salem ML, Nishimura MI, Garrett-Mayer E, Cole DJ, et al. (2009) Increased circulating myeloid-derived suppressor cells correlate with clinical cancer stage, metastatic tumor burden, and doxorubicincyclophosphamide chemotherapy. Cancer Immunol Immunother 58: 49-59.

20. Mikyšková R, Indrová M, Polláková V, Bieblová J, Símová J, et al. (2012) Cyclophosphamide-induced myeloid-derived suppressor cell population is immunosuppressive but not identical to myeloid-derived suppressor cells induced by growing TC-1 tumors. J Immunother 35: 374-384.

21. Sevko A, Sade-Feldman M, Kanterman J, Michels T, Falk CS, et al (2013) Cyclophosphamide promotes chronic inflammation-dependent immunosuppression and prevents antitumor response in melanoma. J Invest Dermatol 133: 1610-1619.
22. Shurin GV, Tourkova IL, Shurin MR (2008) Low-dose chemotherapeutic agents regulate small Rho GTPase activity in dendritic cells. J Immunother 31: 491499.

23. Shurin GV, Tourkova IL, Kaneno R, Shurin MR (2009) Chemotherapeutic agents in noncytotoxic concentrations increase antigen presentation by dendritic cells via an IL-12-dependent mechanism. J Immunol 183: 137-144.

24. Kaneno R, Shurin GV, Tourkova IL, Shurin MR (2009) Chemomodulation of human dendritic cell function by antineoplastic agents in low noncytotoxic concentrations. J Transl Med 7: 58.

25. Zhong H, Gutkin DW, Han B, Ma Y, Keskinov AA, et al. (2014) Origin and pharmacological modulation of tumor-associated regulatory dendritic cells. Int J Cancer.

26. Zhong H, Han B, Tourkova IL, Lokshin A, Rosenbloom A et al. (2007) Low-dose paclitaxel prior to intratumoral dendritic cell vaccine modulates intratumoral cytokine network and lung cancer growth. Clin Cancer Res 13: 5455-5462.

27. Michels T, Shurin GV, Naiditch H, Sevko A, Umansky V, et al. (2012) Paclitaxe promotes differentiation of myeloid-derived suppressor cells into dendritic cells in vitro in a TLR4-independent manner. J Immunotoxicol 9: 292-300.

28. Sevko A, Kremer V, Falk C, Umansky L, Shurin MR, et al. (2012) Application of paclitaxel in low non-cytotoxic doses supports vaccination with melanoma antigens in normal mice. J Immunotoxicol 9: 275-281.

29. Sevko A, Michels T, Vrohlings M, Umansky L, Beckhove P, et al. (2013) Antitumor effect of paclitaxel is mediated by inhibition of myeloid-derived suppressor cells and chronic inflammation in the spontaneous melanoma model. J Immunol 190: 2464-2471. 